\title{
Corrigendum: A Comprehensive Comparison Between the Semi-sterile and Sterile Technique for Closed Reduction and Percutaneous Pinning of Pediatric Supracondylar Humerus Fractures
}

\author{
Guo-Qiang Wang ${ }^{1}$, Qing-Feng Wang ${ }^{2}$ and Xiao-Dong Wang ${ }^{3 *}$ \\ ${ }^{1}$ Children's Hospital of Soochow University, Suzhou, China, ${ }^{2}$ Department of Orthopedic Surgery, People's Hospital of Ningxia \\ Hui Autonomous Region, Yinchuan, China, ${ }^{3}$ Department of Orthopedics, Children's Hospital of Soochow University, Suzhou, \\ China
}

Keywords: pediatric, sterile, fracture, pinning, recovery, infection

\section{A Corrigendum on}

A Comprehensive Comparison Between the Semi-sterile and Sterile Technique for Closed Reduction and Percutaneous Pinning of Pediatric Supracondylar Humerus Fractures

\section{OPEN ACCESS}

Approved by:

Frontiers Editorial Office,

Frontiers Media SA, Switzerland

${ }^{*}$ Correspondence:

Xiao-Dong Wang

xiaodong_wang_chsu@163.com

Specialty section:

This article was submitted to

Pediatric Surgery,

a section of the journal

Frontiers in Surgery

Received: 12 December 2020 Accepted: 14 December 2020

Published: 15 January 2021

Citation:

Wang G-Q, Wang $Q-F$ and Wang X-D

(2021) Corrigendum: A

Comprehensive Comparison Between the Semi-sterile and Sterile Technique

for Closed Reduction and

Percutaneous Pinning of Pediatric

Supracondylar Humerus Fractures.

Front. Surg. 7:640826.

doi: 10.3389/fsurg.2020.640826 by Wang, G.-Q., Wang, Q.-F., and Wang, X.-D. (2020). Front. Surg. 7:594027. doi: $10.3389 /$ fsurg.2020.594027

In the original article, there was an error. ${ }^{* *}$ There is a mistake in the first paragraph of the Method section, where the hospital name that the patients were treated in is incorrect.**

A correction has been made to ${ }^{* *}$ Method $^{* *},{ }^{* *}$ Paragraph 1, first sentence ${ }^{* *}$ :

Original text: Upon approval by the review board, a consecutive retrospective cohort study was conducted on pediatric patients who underwent CRPP for supracondylar humerus fracture over a 3-year period (2017-2019) at Children's Hospital of Soochow University.

CORRECTED text: Upon approval by the review board, a consecutive retrospective cohort study was conducted on pediatric patients who underwent CRPP for supracondylar humerus fracture over a 3-year period (2017-2019) at Children's Hospital of Bao Tou.

In the original article, there was an error. ${ }^{* *}$ There is a mistake in the first paragraph of the Ethics Statement section, where the name of the approval ethical committee is incorrect.**

A correction has been made to ${ }^{* *}$ ETHICS STATEMENT ${ }^{* *},{ }^{* *}$ Paragraph 1 , sentence $1^{* *}$ :

Original text: The studies involving human participants were reviewed and approved by ethical committee of JiangSu province.

CORRECTED text: The studies involving human participants were reviewed and approved by ethical committee of Inner Mongolia Autonomous Region.

The authors apologize for this error and state that this does not change the scientific conclusions of the article in any way. The original article has been updated.

Copyright (c) 2021 Wang, Wang and Wang. This is an open-access article distributed under the terms of the Creative Commons Attribution License (CC BY). The use, distribution or reproduction in other forums is permitted, provided the original author(s) and the copyright owner(s) are credited and that the original publication in this journal is cited, in accordance with accepted academic practice. No use, distribution or reproduction is permitted which does not comply with these terms. 